Cahiers d'études africaines

198-199-200|2010

50 ans

\title{
Au fil des Cahiers
}

Jean-Loup Amselle

\section{(2) OpenEdition}

Journals

Édition électronique

URL : https://journals.openedition.org/etudesafricaines/16151

DOI : 10.4000/etudesafricaines. 16151

ISSN : $1777-5353$

\section{Éditeur}

Éditions de l'EHESS

\section{Édition imprimée}

Date de publication : 20 novembre 2010

Pagination : 375-381

ISBN : 978-2-7132-2252-8

ISSN : 0008-0055

\section{Référence électronique}

Jean-Loup Amselle, «Au fil des Cahiers », Cahiers d'études africaines [En ligne], 198-199-200 | 2010, mis en ligne le 02 janvier 2013, consulté le 23 avril 2022. URL : http://journals.openedition.org/ etudesafricaines/16151; DOI : https://doi.org/10.4000/etudesafricaines.16151

Ce document a été généré automatiquement le 23 avril 2022

(C) Cahiers d'Études africaines 


\title{
Au fil des Cahiers
}

\author{
Jean-Loup Amselle
}

1 Cinquante ans après les indépendances africaines que sont nos amours africaines devenues? Telle est la formule qui pourrait peut-être rendre compte du désarroi dans lequel sont plongés un certain nombre d'africanistes de ma génération, et que je ressens moi-même. L'année 1960, date de la fondation des Cahiers d'Études africaines par Georges Balandier, fournit un bon repère pour cadrer l'évolution de l'africanisme jusqu'à son aboutissement actuel. C'est à la fin des années 1950 et au début des années 1960, en effet, que l'africanisme, sous la houlette de Georges Balandier, prend pleinement son essor avec la fondation en $1958 \mathrm{du}$ «Centre d'études africaines » de la $\mathrm{VI}^{\mathrm{e}}$ section de l'École pratique des hautes études (EPHE, l'ancêtre de l'EHESS) et la création du certificat de sociologie de l'Afrique noire à la Sorbonne en 1962.

2 Je me souviens, à cette époque, avoir déserté l'enseignement poussiéreux dispensé par l'Institut d'ethnologie du Musée de l'Homme pour aller écouter, à la $\mathrm{VI}^{\mathrm{e}}$ section de l'EPHE, Georges Balandier, dont on disait alors qu'il traitait des problèmes de l'Afrique contemporaine, et non d'une Afrique figée dans la tradition. C'était en effet une véritable bouffée d'air mettant l'accent sur le politique, l'histoire et le religieux, il parlait à notre esprit et surtout à notre cœur de militants tiers-mondistes luttant pour l'indépendance de l'Algérie, puis de celles du Vietnam, du Laos et du Cambodge. De ce séminaire de l'EPHE dirigé par Georges Balandier, est issue une bonne partie de l'anthropologie actuelle et surtout la quasi-totalité des africanistes chevronnés. Ce fut un âge d'or de l'africanisme parce que cette spécialité des sciences sociales adossée à une aire culturelle, et non une discipline, correspondait à une période historique précise, celle de l'émancipation du Tiers-Monde et de l'accession des pays africains à l'indépendance. Le continent africain, de même que l'ensemble des pays du Sud, représentait à cette époque un espoir de libération pour la totalité de la planète. Dans ce contexte, les Cahiers offraient une articulation entre engagement politique et pratique scientifique, et il n'est que de feuilleter les premiers numéros de la revue pour s'en assurer. De grands noms prestigieux y figurent: ceux de Fernand Braudel qui présente le premier numéro de la revue, Max Gluckman, Denise Paulme, Paul Mercier, Yves Person, Gilles Sautter, Éric de Dampierre, Georges Niangoran-Bouah, Gilbert 
Rouget, Germaine Dieterlen, Geneviève Calame-Griaule, Immanuel Wallerstein, Aristide Zolberg, Edward Evan Evans-Pritchard, Claude Meillassoux, Roger Bastide, notamment.

3 Cette pléiade de noms prestigieux traduit bien l'impulsion que Georges Balandier, qui avait confié la direction de notre revue à Pierre Alexandre, voulait donner à l'africanisme. Il s'agissait de s'appuyer sur l'anthropologie sociale britannique dans ce qu'elle avait de meilleur : celle d'Evans-Pritchard et de Fortes, bien sûr, mais aussi et surtout celle de Max Gluckman et du Rhodes Livingtone Institute, lequel avait accueilli dans les années 1940-1950 une équipe de chercheurs en sciences sociales renommés : James Clyde Mitchell, William Watson, Arnold Leonard Epstein, Elizabeth Colson, Victor Turner, John Barnes, entre autres. C'est dans le prolongement du Rhodes Livingstone Institute, qui prit plus tard le nom d'École de Manchester, que Balandier voulait situer sa démarche: celle d'une anthropologie politique dynamique axée sur la situation coloniale, l'urbanisation, et la modernité des phénomènes religieux telle qu'elle se manifestait à travers l'étude des messianismes. À cet égard, l'africanisme pouvait apparaître à la fois comme une spécialité adossée à une aire culturelle, mais aussi comme une prise de parti en anthropologie, c'est-à-dire comme la volonté d'en faire une «sociologie ». Il marquait bien, par là, qu'étaient privilégiées l'historicité et la contemporanéité des situations africaines résultant essentiellement de la colonisation. À cette époque, l'islam, l'esclavage et la traite atlantique ne faisaient pas encore partie du domaine d'études de l'africanisme.

4 L'africanisme s'est donc développé contre d'autres courants dominants de l'ethnologie française, représentés par les grands noms de Marcel Griaule, Claude Lévi-Strauss et Louis Dumont. Ce n'est d'ailleurs pas un hasard s'il a servi de marqueur institutionnel, scientifique et politique au sein des sciences sociales traitant des sociétés exotiques, poussant quelques-uns des premiers anthropologues formés par Balandier à rejoindre ultérieurement certains de ses confrères. Il faudrait montrer en quoi l'engagement intellectuel et politique de certains anthropologues, africanistes ou non, marqués par le marxisme et l'appartenance au Parti communiste a pris ultérieurement la forme d'une conversion au primitivisme. Ce processus, qui trouve des prolongements actuels, éclaire d'ailleurs également les impasses d'une fraction du monde politique actuel incapable de sortir des schémas évolutionnistes d'antan autrement que par un retour à un passé supposé des sociétés exotiques. Pour cette raison, il ne s'agit donc pas uniquement d' « une autre histoire » puisque cela montre amplement que ce qui s'est joué au tournant des années 1960 autour de la personne de Georges Balandier, du Centre d'études africaines et des Cahiers a eu une résonance beaucoup plus forte que la seule prise en compte de l'œuvre d'un africaniste ou de celle d'un centre de recherches et d'une revue qui lui furent associés.

5 Le début des années 1960 est l'époque du lancement, par Georges Balandier et Gilles Sautter, de missions pluridisciplinaires d'enquête sur le terrain effectuées par de jeunes chercheurs dans le cadre de la thématique des "terroirs" et des "communautés rurales » africaines ${ }^{1}$. Un certain nombre de comptes rendus de ces missions figure ainsi en bonne place dans les Cahiers, celles d'Ariane Deluz et Claude Meillassoux chez les Gouro de Côte-d'Ivoire ${ }^{2}$, de Michel Cartry et Gérard Remy chez les Gourmantché de Haute-Volta ${ }^{3}$, d'Alfred Adler chez les Mbay du Tchad, etc. ${ }^{4}$. De ces missions, sortira un article qui sera à l'origine de tout un courant de recherches d'anthropologie économique, l'«Essai d'interprétation du phénomène économique dans les sociétés traditionnelles d'auto-subsistance » de Claude Meillassoux ${ }^{5}$. 
6 En 1966, c'est au tour du Centre d'analyse et de recherche documentaires pour l'Afrique noire (CARDAN), créé en 1959 et pilier du Centre d'études africaines d'alors, de faire l'objet d'une présentation en forme de bilan, au terme de ses cinq premières années d'existence ${ }^{6}$. Rédigé par Françoise Héritier et René Bureau, ce rapport présente les activités d'un centre dont l'existence avait certainement un sens au début des années 1960 puisqu'il était alors possible d'offrir un panorama de l'ensemble de la littérature, des institutions de recherche et des chercheurs œuvrant dans le domaine africaniste. Mais ce centre était sans doute voué à disparaitre, comme ce fut effectivement le cas, avec la prolifération de la documentation relative à l'Afrique au sud du Sahara dans les dernières décennies du siècle précédent.

$7 \mathrm{Au}$ cours des années suivantes, on peut remarquer dans les Cahiers, outre quelques articles qui feront date comme "If the Dogon » de Mary Douglas ${ }^{7}$, la restitution de travaux d'équipes de recherches ayant durablement marqué le champ de l'africanisme, comme celle œuvrant sur la littérature orale, et centrée autour de Denise Paulme ${ }^{8}$. C'est également le cas de celle traitant des "relations de dépendance personnelle", armature essentielle de l'anthropologie politique développée à la même époque par Georges Balandier', et dont on trouve un prolongement dans le numéro sur ce même thème coordonné par Pierre Alexandre au début des années $1980^{10}$. C'est à ce momentphare des études africanistes qu'est consacré le bilan et l'état des lieux du «Centre d'études africaines " publié en 1972 dans les Cahiers ${ }^{11}$.

8 Les années 1970-1980 marquent toutefois une inflexion dans le champ des études africanistes, inflexion dont on trouve un écho dans les Cahiers. De nouveaux thèmes apparaissent comme l'histoire ${ }^{12}$, les femmes ${ }^{13}$, l'écologie ${ }^{14}$, la santé ${ }^{15}$ et l'État contemporain ${ }^{16}$, même si des thèmes plus classiquement installés quoique modernes, comme les villes et les situations urbaines, continuent de mobiliser les rédacteurs de la revue $^{17}$.

9 Les années 1990 voient s'accentuer les modifications de la ligne éditoriale avec le départ de Pierre Alexandre en 1987 et son remplacement par l'auteur des présentes lignes. Il s'agit tout d'abord d'une nouveauté thématique marquée par la confection de numéros consacrés à des sujets qui n'avaient jamais été abordés par la revue comme la mémoire ${ }^{18}$, la «malédiction » du continent africain ${ }^{19}$, l'entreprise ${ }^{20}$, la démocratie ${ }^{21}$, les littératures ${ }^{22}$, les images ${ }^{23}$, la violence ${ }^{24}$, les musée ${ }^{25}$ ou bien encore de ceux centrés sur certaines ethnies ou aires culturelles spécifiques (numéros manding, peul, Corne de l'Afrique ${ }^{26}$. Mais la grande nouveauté réside dans l'ouverture des Cahiers au Maghreb et à ce que l'on a désormais coutume de nommer les « diasporas noires des Amériques » («Le Savant et le Berbère. Mesurer la différence » ${ }^{27}$, «Les identités africaines au Brésil $»^{28}$, « La Caraïbe $»^{29}$.

10 Cet effort de renouvellement et d'ouverture se poursuit au cours des années 2000 avec des numéros spéciaux sur les langues ${ }^{30}$, l'enseignement ${ }^{31}$, le postcolonialisme ${ }^{32}$, l'esclavage $^{33}$, les femmes ${ }^{34}$, le tourisme $e^{35}$. Ce qui retient également l'attention est la reprise de questions classiques de l'anthropologie comme celles du politique ${ }^{36}$, des parentés à plaisanterie ${ }^{37}$ ou de la sorcellerie ${ }^{38}$ appréhendées cette fois sous l'angle du contemporain. Les numéros prévus ou en préparation sur l'islam ou le développement prolongent l'inscription de notre revue dans le cadre de cette nouvelle problématique.

11 Ce changement dans les thèmes abordés par les Cahiers, indépendamment des nombreux articles publiés dans les numéros de mélanges sans lesquels une revue ne serait pas digne de ce nom, s'est accompagné d'un profond renouvellement de son 
Comité de rédaction. Du petit noyau fondateur ne subsistent que Georges Balandier et Joseph Tubiana. D'autres noms ayant joué un rôle majeur dans la vie de la revue et de l'institution qui l'héberge, l'EHESs - comme ceux de Marc Augé et d'Emmanuel Terray ont désormais rejoint l'aréopage que constitue le Comité de direction ${ }^{39}$. Certains nouveaux critères et au premier chef celui de la parité ont fait l'objet d'une attention particulière : sur les dix-neuf membres que comporte le Comité de rédaction, six sont des femmes, mais ce nombre est insuffisant, de même que celui des collègues africains.

Ce constat nous ramène à la question que je posais au début: cinquante ans après les indépendances africaines que sont nos amours africaines devenues? Quel est l'avenir de l'africanisme alors que celui-ci est désormais contesté par les postcoloniaux en tant que spécialité pratiquée par des « Blancs » sur une réalité africaine "noire »? Quel est l'avenir des Cahiers alors que de multiples revues africanistes ont vu le jour depuis 1960 et que la politologie, en particulier, a accaparé en grande partie l'attention portée à ce continent? Peut-on être encore africaniste comme on l'était dans les années 1960, en s'appuyant exclusivement ou principalement sur l'anthropologie et sur l'histoire? L'africanisme n'a-t-il pas changé radicalement de sens avec la globalisation, les migrations, la disparition de la coupure profonde entre l'ici et l'ailleurs et ce qui en découle, c'est-à-dire la nécessité de plus en plus affirmée de pratiquer des enquêtes éclatées ou multisituées?

Cette nouvelle donne ou ces nouveaux requisits de la recherche africaniste ont certes trouvé un écho dans les pages de notre revue mais il n'est pas douteux qu'ils représentent un défi pour les recherches du type "aires culturelles ", qui demeurent un des piliers de la politique scientifique de l'EHESS ou d'autres institutions françaises.

En somme, peut-on être encore africaniste et fier de l'être ? Cette interrogation renvoie à son tour à la représentation de l'Afrique dans l'imaginaire occidental. Le continent africain représentait, dans les années 1960, on l'a dit, un espoir de libération pour l'ensemble de la planète. Le fait qu'elle soit « mal partie », pour reprendre l'expression de René Dumont, ou pas partie du tout, que son poids économique et son statut politique occupent une place marginale dans le concert des nations et dans notre monde économique globalisé, a poussé certains africanistes renommés à abandonner ce champ d'études pour des continents plus attrayants ou pour des réflexions plus théoriques, c'est-à-dire moins enracinées dans un terrain d'enquête particulier. Cette déception suivie d'une désertion est d'ailleurs sans doute largement liée à l'engagement politique et à l'implication de ces chercheurs dans la contemporanéité du continent africain, puisqu'elle ne semble pas concerner au même titre les chercheurs travaillant sur d'autres continents ethnologiques.

Ce constat renverrait sans doute également au caractère historiquement et politiquement «chaud» de l'Afrique par rapport à d'autres terrains plus «froids » comme ceux qui furent l'objet de l'attention et de l'affection des anthropologues structuralistes, au premier rang desquels se trouve Claude Lévi-Strauss. On ne peut qu'être frappé, à cet égard, par le « trou noir » que représente l'Afrique dans l'œuvre de ce chercheur prestigieux, comme si l'historicité de l'Afrique, dans ses meilleurs comme dans ses pires aspects, l'avait convaincu de passer son chemin. Toujours est-il que c'est sans doute parce qu'ils considéraient que l'Afrique avait mal «tourné », qu'elle n'avait pas répondu aux espoirs placés en elle, que certains africanistes, en amoureux déçus, s'en sont détournés. Mais cette déception et cet abandon sont-ils justifiés? Les maux imputés à l'Afrique lui sont-ils propres ? La corruption et le népotisme ne sont-ils pas la 
chose du monde la mieux partagée au point qu'ils font parfois étrangement ressembler notre pays à certaines monarchies pétrolières du Golfe de Guinée ?

En d'autres termes, plus que comme repoussoir, ne convient-il pas de concevoir l'Afrique comme un miroir ou un analyseur de notre propre modernité, au sens le plus global de ce terme. L'Afrique ne détient-elle pas le secret du pouvoir? Non pas d'un pouvoir démocratique idéal, celui des droits de l'homme dont on a de plus en plus de mal à discerner les effets, mais celui du pouvoir effectif, celui de la "guerre des races " dont Michel Foucault dans Il faut défendre la sociétét ${ }^{40}$ a admirablement retracé la généalogie. L'auteur de Surveiller et puniri ${ }^{41}$, qui associait ce modèle à Jérusalem par opposition à celui de la philosophie politique qu'il liait à Rome, était loin de se douter que ce schème valait également pour l'Afrique. Pourtant, l'opposition binaire " gens du pouvoir-gens de la terre ", opposition présente à la fois dans l'ensemble des systèmes politiques africains précoloniaux et dans l'historiographie de la France et de l'Angleterre sous la forme de la " guerre des deux races ", semble bien fournir une sorte de matrice commune permettant de transcender les différences dites culturelles ${ }^{42}$. Bref, ce qui a motivé la constitution du champ d'études africanistes, de l'« aire culturelle » africaine, et donc la création du "Centre d'études africaines » ainsi que des Cahiers d'Études africaines, soit la perception d'une différence radicale entre l'Europe et l'Afrique, pourrait bien être au contraire l'espace d'un lieu commun. Plus qu'à l'étrangeté de l'Afrique, c'est à la familiarité que nous entretenons avec elle que l'on devrait s'attacher. Et en cela, la globalisation actuelle, et ce qui l'accompagne, c'est-àdire la compénétration de l'ensemble des cultures du monde, ne serait-elle pas le moyen de faire retour sur le "grand partage " entre l'Afrique et l'Europe, sur l'institution d'une césure radicale entre ces deux éléments, et sur la nécessité de la dépasser?

\section{NOTES}

1. Un colloque présentant le bilan des actions menées autour de cette thématique sera organisé en 1972 à Abidjan dans le cadre de l'ORSTOM (actuel IRD) et donnera lieu à une publication effectuée sous ma responsabilité (Communautés rurales et paysanneries tropicales, Travaux et documents de l'ORSTOM, Paris, 1976.)
2. $\mathrm{N}^{\circ} 2,1960$.
3. $\mathrm{N}^{\circ} 11,1963$.
4. $\mathrm{N}^{\circ} 18,1965$.
5. $\mathrm{N}^{\circ} 4,1960$.
6. $\mathrm{N}^{\circ} 21,1966$.
7. $\mathrm{N}^{\circ} 28,1967$.
8. $N^{\circ} 30,1968 ; n^{\circ} 45,1972 ; n^{\circ} 60,1975 ; n^{\circ} 73-76,1979$.
9. $\mathrm{N}^{\circ} 35,1969$. 
10. $\mathrm{N}^{\circ} 87-88,1982$.

11. $\mathrm{N}^{0} 48,1972$.

12. No 61-62, $1976 ; n^{\circ} 105-106,1987$.

13. $\mathrm{N}^{\circ} 65,1977$.

14. $\mathrm{N}^{\circ} 77-78,1980 ; \mathrm{n}^{\circ} 101-102,1986 ; \mathrm{n}^{\circ} 114,1989$.

15. $\mathrm{N}^{\circ} 85-86,1982$.

16. $\mathrm{N}^{\circ} 69-70,1978 ; \mathrm{n}^{\circ} 103,1986$.

17. $\mathrm{N}^{\circ} 51,1973 ; \mathrm{n}^{\circ} 99,1985$.

18. $\mathrm{N}^{\circ} 107-108,1987 ; \mathrm{n}^{\circ} 119,1990$.

19. № $121-122,1991$.

20. $\mathrm{N}^{\mathrm{o}} 124,1991$.

21. $\mathrm{N}^{\mathrm{o}} 137,1995$.

22. $\mathrm{N}^{\circ} 140,1995$.

23. $\mathrm{N}^{\circ}$ 141-142, 1996.

24. $\mathrm{N}^{\circ} 150-152,1998$.

25. No $155-156,1999$.

26. $\mathrm{N}^{\circ} 111-112,1988 ; \mathrm{n}^{\circ} 133-135,1994 ; \mathrm{n}^{\circ} 144,1996 ; \mathrm{n}^{\circ} 146,1997$.

27. $\mathrm{N}^{\mathrm{o}} 129,1993$.

28. $\mathrm{N}^{\mathrm{o}} 125,1992$.

29. $\mathrm{N}^{\circ} 148,1997$.

30. $\mathrm{N}^{\circ}$ 163-164, 2001.

31. $\mathrm{N}^{\mathrm{o}} 169-170,2003$.

32. $\mathrm{N}^{\mathrm{o}}$ 173-174, 2004.

33. $\mathrm{N}^{\circ}$ 179-180, 2005.

34. $\mathrm{N}^{\circ}$ 187-188, 2007.

35. $\mathrm{N}^{\mathrm{o}}$ 193-194, 2009.

36. $\mathrm{N}^{\mathrm{o}} 178,2005$.

37. $\mathrm{N}^{\circ} 184,2006$.

38. $\mathrm{N}^{\circ}$ 189-190, 2008.

39. À cela, il faudrait ajouter toutes celles et ceux sans lesquels notre revue n'aurait pu paraître: Annick Le Pape, Christine Messiant, Cléo Pace, Yvette Trabut, Christian Campo, Raphaël Jacquet, sans oublier les secrétaires de rédaction actuelles qui ont œuvré à ce numéro : Nadège Chabloz et Martine Morier.

40. (Cours au Collège de France, 1976), Paris, Gallimard-Seuil (« Hautes études »), 1997.

41. Paris, Gallimard (« Tel »), 1975.

42. Voir dans ce numéro, l'article de Jean-Loup AMSELLE, «L'Afrique peut-elle être décrochée de l'Occident?». 


\section{AUTEUR}

JEAN-LOUP AMSELLE

École des hautes études en sciences sociales, Paris. 